\title{
Evaluation of The Mechanical Properties of The Multilayered Films by Nanoindentation at a Penetration Depth as small as $10 \mathrm{~nm}$
}

\author{
Takeshi SAWA, Atsushi SHIMAMOTO and Kohichi TANAKA
}

Department of Mechanical Engineering, Nagaoka University of Technology, 1603-1 Kamitomioka, Nagaoka, Niigata, 940-21 JAPAN

Abstract - In nanometer order nanoindentation test, roundness or truncation of the indenter tip cannot be avoided. In this paper, we analyzed the indentation problem of a rounded triangular indentation into a layered elastic half-space by a finite element analysis, and then established a method to estimate the intrinsic elastic modulus of the film from the nanoindentation data. The method was demonstrated by the nanoindentation test with a less than $10 \mathrm{~nm}$ penetration depth on a $10 \mathrm{~mm}$ thick diamond-like carbon film deposited on a $50 \mathrm{~nm}$ thick magnetic layer.

Keywords : Berkovich indenter, tip truncation, composite Young's modulus, hard disk, diamond-likecarbon thin film.

\section{INTRODUCTION}

The media of hard disk drives are composed of multilayered films. The magnetic layer is protected by carbon overcoat, of which thickness in recent media becomes less than $30 \mathrm{~nm}$ for improving the recording density of hard disks. In parallel with this, the flying height of the head slider on the disk decreases to less than $50 \mathrm{~nm}$, and the proximity contact recording will become reality in very near future. The tribology at the Head/Disk Interface (HDI) will be crucial in designing the future hard disk drives. Thus, the establishment of the method to evaluate the mechanical properties of $10 \mathrm{~nm}$ order thickness carbon films on a multilayer structure is urgently needed.

Nanoindentation is a simple and effective means for evaluating the mechanical properties of thin films. In the method, the applied load, $P$, and the penetration depth, $h$, of the indenter are continuously monitored, and the mechanical properties are determined from the obtained $P$ - $h$ relationship. In common sense, for evaluating the intrinsic mechanical properties of a thin film. the penetration depth should not exceed one tenth of the film thickness.

Doerner and Nix [1] suggested that the effective composite Young's modulus, $E_{e f f}^{*}$, in a layered solid can be described as a function of plain strain Young's moduli, $1 / E^{*}=E /\left(1-\nu^{2}\right)$, of the relevant materials;

$$
\frac{1}{E_{e f f}^{*}}=\frac{1}{E_{f}^{*}}\left(1-e^{-\beta t / h}\right)+\frac{1}{E_{s}^{*}} \epsilon^{-\beta t / h}+\frac{1}{E_{I}^{*}}
$$

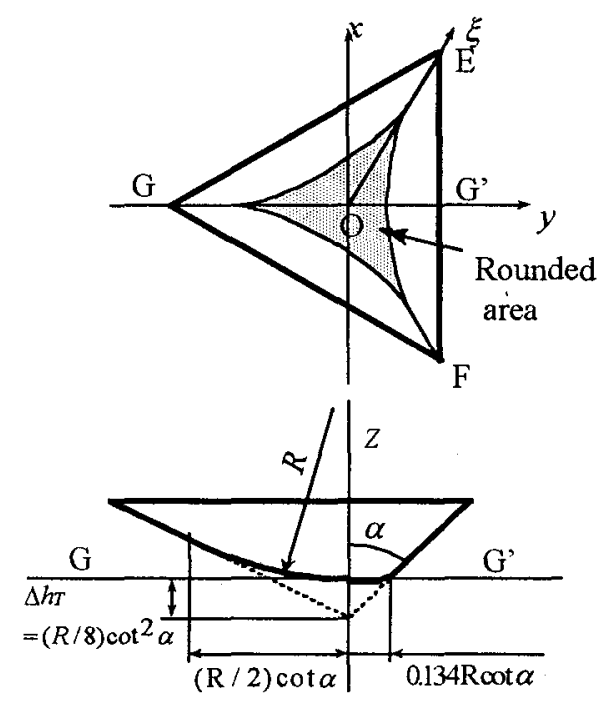

Figure 1: Geometrical illustration of a truncated Berkovich indenter.

where $\beta$ is an unknown parameter, $t$ the film thickness, $E$ the Young's modulus, and $\nu$ Poisson's ratio. Subscripts $f, s$ and $I$ refer to the film, substrate, and indenter properties, respectively. They determined $\beta$ based on measurements of tungsten films on silicon substrates. King [2] analyzed the problem of triangular punches indenting a layered solid via a singular integral equation, and numerically obtained $\beta$ as a function of normalized contact radius by $t$.

A triangular indenter is commonly used for nanoindentation measurement for the reason that the three facets theoretically converge to a single point. However, in practice, the truncation or roundness of the indenter tip may not be avoided during machining and testing, and the truncation may have a critical influence on nanometer order indentation testing. Namely, the problem in a nanoindentation test is not only the performance of a nanoindentation instrument but also the effect of the roundness or truncation of the indenter tip.

Shimamoto et al. [3] analyzed the effect of the tip truncation of a Berkovich indenter when indenting an elastic halfspace by a three-dimensional elastic finite el- 


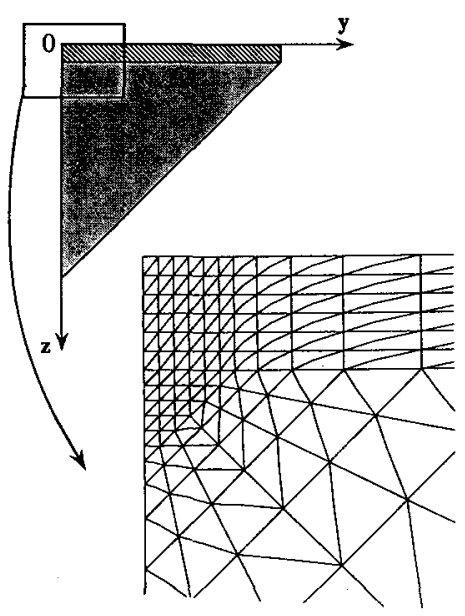

Figure 2: The finite element mesh of the layered solid.

ement method (FEM). They modeled the geometry of the tip truncation as shown in Fig. 1. The radius of curvature at the apex, $R$, is defined as represented in the G-G'section, where the profile of indenter is tangential to the edge $O G$ and its center is situated on the indentation axis ( $z$ direction). They suggested that the $P-h$ relationship can be approximately written by

$$
P+\Delta P=C(\tan \alpha) E^{*}(h+\Delta h)^{2},
$$

where $C$ is a geometrical factor and $\alpha$ the apical angle of the indenter. They determined that the factor $C$ is 0.95 for the Berkovich indenter with $\alpha=65^{\circ} . \Delta h$ and $\Delta P$ are related to the truncation length, $\Delta h_{T}=$ $(R / 8) \cot ^{2} \alpha$, and expressed by

$$
\begin{aligned}
\Delta h & =1.10 \Delta h_{T}, \\
\Delta P & =1.16 C(\tan \alpha) E^{*} \Delta h_{T} .
\end{aligned}
$$

In this paper, we analyze the process of a rounded Berkovich indenter pressing into an elastic layered solid by FEM, and then demonstrate a method which can be utilized to calculate the intrinsic elastic modulus of a diamond-like carbon film with a thickness of $10 \mathrm{~nm}$.

\section{FINITE ELEMENT ANALYSIS}

Figure 2 shows the finite element mesh of an elastic layered solid. For treating the coating, six mesh layers with a minimum thickness of $2 \mathrm{~nm}$ are placed onto the previous mesh of a non-layered solid [3][4]. The elements are ten-noded isoparametric blocks (threedimensional tetrahedral). The total number of nodes and elements are 6396 and 4014 , respectively. The dimension of the analytical region is a tetrahedron with $2347 \mathrm{~nm}$ in sides along to the $x, y$ and $z$ axes. The minimum-sized elements are placed along to $x, y$ and

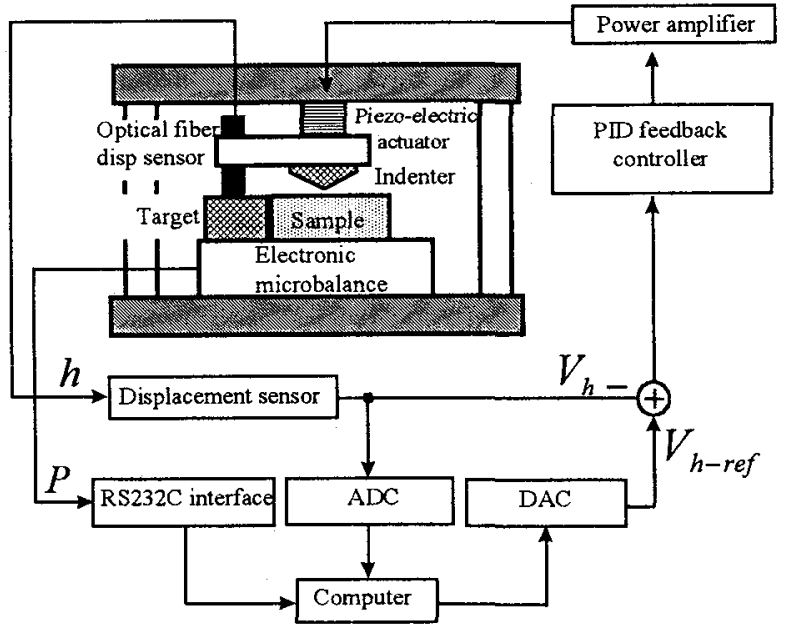

Figure 3: Schematic block diagram of the depth controlling nanoindentation tester.

$z$ axes beneath the origin $\mathrm{O}$ shown in Fig. 2. The displacements at the outer boundaries are fixed to be zero. The FEM analyses were computed for the indentation in a 30nm-thickness film by using a rigid indenter $\left(1 / E_{I}^{*}=0\right)$. Young's modulus of the substrate $E_{s}$ was fixed to be $206 \mathrm{GPa}$ and that of the film was varied into four levels as $E_{f} / E_{s}=1 / 4,1 / 2,1,2,4$, equating Poisson's ratio of all the materials to 0.3 . The radius curvature of the indenter was varied into four leveis, $R=0,100,200$ and $300 \mathrm{~nm}$.

\section{NANOINDENTATION EXPERIMENT}

A $10 \mathrm{~nm}$ thick diamond-like carbon (DLC) thin film was deposited on a $50 \mathrm{~nm}$ thick CoCrTa coating presputtered on a toughened glass substrate.

The nanoindentation tests were performed by a depth controlling nanoindentation tester [5] as shown in Fig. 3 under a penetration speed of $0.25 \mathrm{~nm}$ using a Berkovich indenter [5]. The applied load of the indenter is measured by an electronic microbalance with a $0.1 \mu \mathrm{N}$ resolution and the relative displacement between the indenter and the sample surface is monitored by an optical fiber displacement sensor [6] with a $0.1 \mathrm{~nm}$ resolution and $100 \mathrm{~Hz}$ bandwidth. The indenter and the displacement sensor probe are fixed to an aluminum block, and the block is driven by a piezo-electric actuator with a $15 \mu \mathrm{m}$ travel length. The extension of the actuator is servo-controlled by a power amplifier via a proportional-integral-derivative (PID) controller to compensate difference between output signal from the displacement sensor, $V_{h}$, and reference signal, $V_{h-r e f}$, from a digital-to-analog converter (DAC). The computer in Fig. 3 controls $V_{h-r e f}$ and stores $P$ and $V_{h}$ continuously through the RS-232C interface and an analogto-digital converter $(\mathrm{ADC})$, respectively. 


\section{NUMERICAL RESULTS}

Figure 4 shows $E_{\text {eff }}^{*} / E_{s}^{*}$ vs $h / t$ relationships obtained by the FEM calculation for the films with $E_{f} / E_{s}=4$ and $1 / 4$; the results for differently truncated indenters are indicated by using different symbols, while those in the case of indenting on films with relative stiffnesses other than 4 and $1 / 4$ are not shown, but behave simillar on the semi-log plot. The result of a non-truncated indenter $(R=0)$ indenting on films with four different stiffnesses are represented in Fig. 4 by the curves originated from $E_{f} / E_{s}$ at $h / t=0$, which were obtained by the regression of the calculated data; the corresponding result at $E_{f} / E_{s}=1$ converges to a straight line at $E_{\text {eff }}^{*} / E_{s}^{*}=1$. These curves for the non-truncated indenter were approximated to eqn (1) when $E_{f}<E_{s}$, while to the following equation when $E_{f}>E_{s}$,

$$
\frac{1}{E_{\text {eff }}^{*}}=\frac{1}{E_{s}^{*}}\left(1-e^{-\gamma(h / t)}\right)+\frac{1}{E_{f}^{*}} e^{-\gamma(h / t)}+\frac{1}{E_{I}^{*}},
$$

where $\gamma$ is an unknown parameter. Both $\beta$ and $\gamma$ are weakly dependent on the $h / t$ and the regressed curves gave the best fitting to the approximation when

$$
\begin{aligned}
& \beta=0.257+0.022(h / t)\left(\text { for } E_{f} / E_{s}=1 / 4\right), \\
& \beta=0.261+0.094(h / t)\left(\text { for } E_{f} / E_{s}=1 / 2\right), \\
& \gamma=1.277-0.293(h / t)\left(\text { for } E_{f} / E_{s}=2\right), \\
& \gamma=1.013-0.180(h / t)\left(\text { for } E_{f} / E_{s}=4\right) .
\end{aligned}
$$

The $E_{e f f}^{*} / E_{s}^{*}$ values for the truncated indenter become higher than those for the non-truncated indenter and the discrepancy of the values between the two indenters increases with decreasing in $h / t$. Particularly, a striking discrepancy appears when $E_{f}<E_{s}$; the values for the truncated indenter increase with decreasing in $h / t$, while the trend appears converse for the nontruncated indenter. These facts suggest the opposite of the commonsense view that the intrinsic mechanical properties of a thin film are measured with the penetration depth less than one tenth of the film thickness.

Figure 5 shows the replot of Fig. 4 replacing $h$ by $h+\Delta h$ and $P$ by $P+\Delta P$, where $\Delta h$ and $\Delta P$ are given in eqns (3). It is obvious that effect of truncation on $E_{\text {eff }}^{*} / E_{s}^{*}$ is corrected by this replot based on the modified $P-h$ relation in eqn (2).

\section{EXPERIMENTAL RESULTS AND DISCUSSION}

In this study, making use of Fig. 5 on the basis of the relations in eqns (4) and (5), we estimate the elastic modulus of a $10 \mathrm{~nm}$ thick DLC layer on top of a $50 \mathrm{~nm}$ thick CoCrTa magnetic coating deposited on a

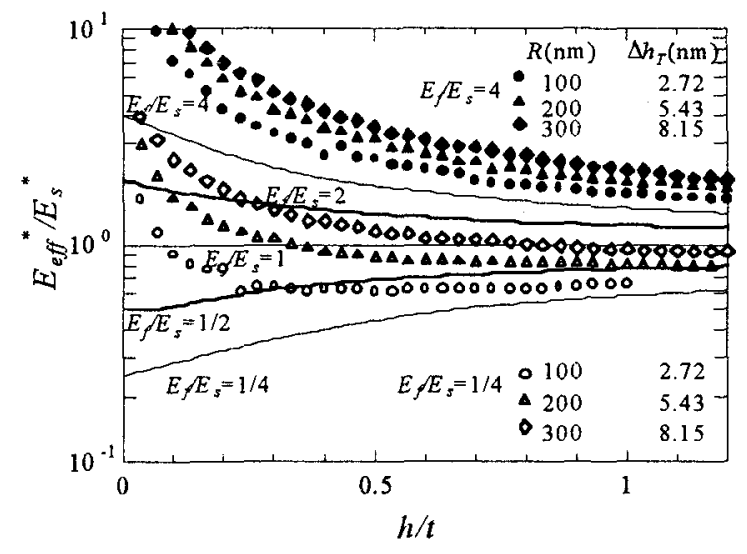

Figure 4: Relationship between $E_{\text {eff }}^{*} / E_{s}^{*}$ and $h / t$ for truncated Berkovich indenters calculated by FEM.

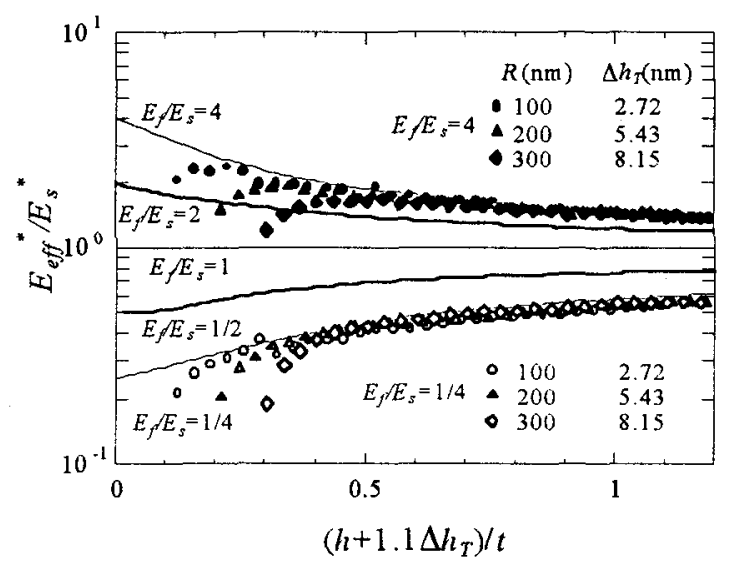

Figure 5: Replot of Fig. 4 replacing $h$ by $h+\Delta h$ and $P$ by $P+\triangle P$.

glass substrate. For this, we should determine $\Delta h_{T}$ for the indenter to be used and $E_{s}^{*}$ for CoCrTa magnetic coating/glass system. For the determination of $\Delta h_{T}$ for the indenter, we follow the procedure mentioned in a previous paper [3]. We performed nanoindentation up to $h=8 \mathrm{~nm}$ on a substrate glass (Corning code 7059F) having $E=67.5 \mathrm{GPa}$ and $\nu=0.28$. We obtained a loading-unloading curve with no hysteresis and confirmed its elastic behavior by reloading. Then, we analyzed the elastic loading curve thus obtained as to give a best-fitting to the $P-h$ relationship in eqn (2) and determined at $\Delta h_{T}=2.2 \mathrm{~nm}$ by taking eqn (3) into account.

Figure 6 shows $P$-h curves for the $\mathrm{CoCrTa} /$ glass coating system with and without a DLC coating. We can determine $E_{s}^{*}$ by analysing the stiffness-measuring technique [1] to the unloading curve of the $\mathrm{CoCrTa}$ /glass coating system without a DLC coating, of which load- 


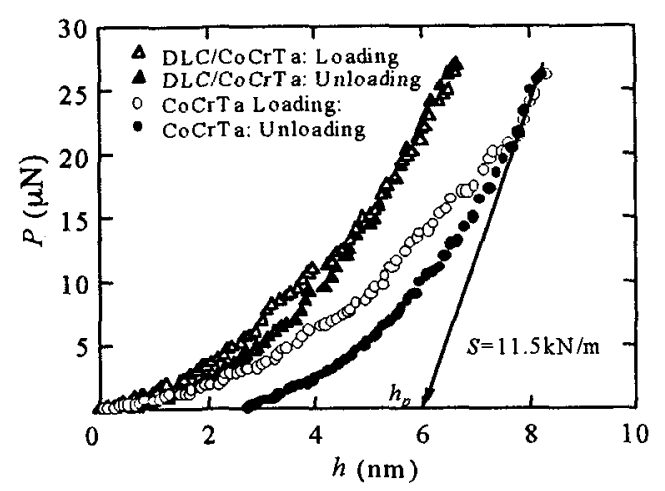

Figure 6: $P$ - $h$ curves for a CoCrTa/glass system with and without a DLC coating. The thicknesses of the DLC and CoCrTa layers were 10 and $50 \mathrm{~nm}$, respectively.

ing cycle shows a hysteresis indicating the occurrence of plastic deformation. Assuming that the contact area remains constant during initial unloading stage, an approximated elastic contact solution can be obtained by using the flat-ended punch model with equivalent contact area [1]. The solution can be written by

$$
S=C_{A} E^{*} \sqrt{A_{r}},
$$

where $C_{A}$ is a geometrical factor and $A_{r}$ the real projected contact area. $C_{A}$ for Berkovich indenter is evaluated at 1.46 for elastic contact [3] and 1.17 for elasticplastic or plastic contact [2].

The indentation curve of the CoCrTa layer shows elastic-plastic behavior but not far from elastic. Thus we assume that the behavior represents a transient phase from elastic to elastic-plastic and that $C_{A}$ becomes the arithmetic mean of the pertinent values; $(1.46+1.17) / 2=1.32$. According to the assumption by Doerner and Nix [1], $A_{r}$ may be related to the plastic depth $h_{p}$ indicated in Fig. 6 and calculated by considering the geometry of the tip-truncated Berkovich indenter;

$$
A_{r}=3 \sqrt{3}\left(\tan ^{2} \alpha\right)\left(h_{p}+\Delta h_{T}\right)^{2} .
$$

The contact stiffness, $S$, and the plastic depth, $h_{p}$, at the maximum load for the CoCrTa layer are estimated at $11.5 \mathrm{kN} / \mathrm{m}$ and $6.0 \mathrm{~nm}$, respectively. Substituting eqn (7) into eqn (6), $E^{*}$ yields $219 \mathrm{GPa}$. This value may be reasonable because it is close to the composite elastic modulus calculated from a bulk cobalt.

The $P$ - $h$ curve for the DLC film behaves more elastic because the hysteresis is not as clear as the previous case. Figure 7 shows the $E_{\text {eff }}^{*} / E_{s}^{*}$ vs $h / t$ relationship for the experimental data of the DLC layer. $E_{\text {eff }}^{*}$ is calculated from eqn (2) by assuming that $E_{I}=1050 \mathrm{GPa}$

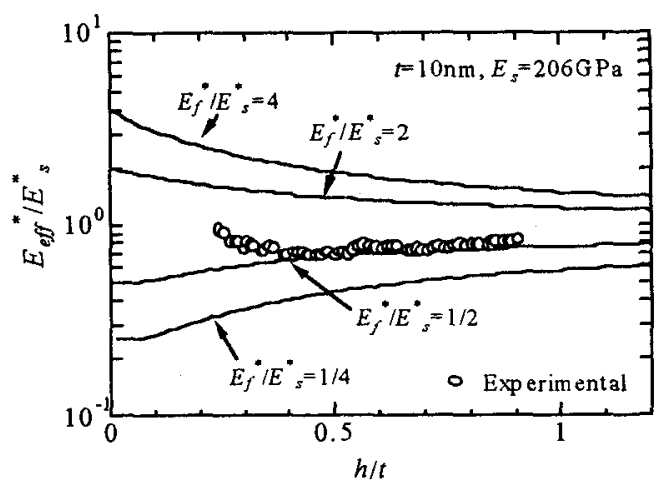

Figure 7: Experimental $E_{\text {eff }}^{*} / E_{s}^{*}$ vs $(h+\Delta h) / t$ relationship for a DLC film of $10 \mathrm{~nm}$ thickness onto CoCrTa layer of $50 \mathrm{~nm}$ thickness.

and $\nu=0.1$. The experimental data agree with the curve for $E_{e f f}^{*} / E_{s}^{*}=1 / 2$, and the intrinsic composite elastic modulus of the DLC film can be estimated at $114 \mathrm{GPa}$. This value is comparable order of the elastic modulus prepared by a plasma decomposition method obtained by Jiang et al. [7]. The Young's moduli which they obtained decreased from $220 \mathrm{GPa}$ to $90 \mathrm{MPa}$ with the increase in $\mathrm{C}_{2} \mathrm{H}_{2}$ gas pressure.

In conclusion, this method is useful to evaluate the elastic moduli of films as thin as $10 \mathrm{~nm}$ which will be used as a protective layer for information storage systems in the near future.

It is acknowledged that this study was partially supported by the Storage Research Consortium (SRC).

\section{References}

[1] M. F. Doerner and W. D. Nix, J. Mater. Res., 1(4), 601 (1986).

[2] R. B. King, Int. J. Solids Structures, 23(12), 1657 (1987).

[3] A. Shimamoto, K. Tanaka, Y. Akiyama and H. Yoshizaki, Phil. Mag. A, 74(5), 1097 (1996).

[4] Y. Murakami, K. Tanaka, M. Itokazu and A. Shimamoto, Phil. Mag. A, 69(6), 1131 (1994).

[5] A. Shimamoto and K. Tanaka, Rev. Sci. Instrum., 68(9), 3494 (1997).

[6] A. Shimamoto and K. Tanaka, Appl. Opt., 34(25), 5854 (1995).

[7] X. Jiang, K. Reichelt and B. Stritzer, J. Appl. Phys., 68(3), 1018 (1990). 